\title{
Propuestas para remover la arquitectura de la exclusión educativa
}

Enviado: 1 de marzo de 2020 / Aceptado: 5 de abril de 2020

Publicado: 12 de julio de 2020

COVADONGA MENÉNDEZ SUÁREZ

Servicio de Orientación Educativa y Formación del Profesorado, Consejería de Educación y Cultura del Principado de Asturias, España.

covadongms@educastur.org

EDUARDO DOPICO

Dpto. CCEE,

Universidad de Oviedo, España.

dopicoeduardo@uniovi.es

\section{RESUMEN}

Tratando de avanzar hacia un sistema educativo inclusivo, en esta investigación se ahonda en las percepciones del colectivo docente sobre la educación inclusiva como primer paso para construir sistemas educativos que aúnen calidad con equidad. Paralelamente, se analiza la normativa reguladora de respuesta a la diversidad de la administración educativa de Asturias (España), para comprobar si el discurso inclusivo se acompaña de políticas y prácticas congruentes contra la exclusión educativa. Para recabar esta información se crearon grupos de discusión online en los que participaron 41 profesoras de las especialidades de Audición y Lenguaje y Pedagogía Terapéutica y se pasó un cuestionario a 299 docentes generalistas de las etapas de Educación Secundaria Obligatoria y Formación Profesional. El diseño metodológico de la investigación concluyó con un análisis documental de la

\section{ABSTRACT}

Proposals to remove the architecture of educational exclusion

Trying to move towards an inclusive education system, this investigation delves into teachers' perceptions of inclusive education as a previous step to build educational systems that combine quality with equity. In parallel, the regulatory regulation response to the diversity by the educational administration of Asturias (Spain) is analyzed, to check if inclusive discourse is accompanied by congruent policies and practices against educational exclusion. To gather this information, online discussion groups were created in which 41 teachers from the specialties of Hearing and Language and Therapeutic Pedagogy participated and a questionnaire was passed to 299 general teachers in the stages of Compulsory Secondary Education and Vocational Training. The methodological design of the research concluded with 
normativa reguladora de la administración educativa en materia de inclusión. De los resultados obtenidos, parece probado que el concepto de inclusión sigue sin comprenderse en los centros ordinarios. Además, el profesorado considera que no posee la formación adecuada para responder a la diversidad, pone en duda el derecho de todo el alumnado a escolarizarse en colegios comunes y apoya el mantenimiento de centros de educación especial. Por su parte, del análisis de los procedimientos reguladores de la administración, se desprende falta de coherencia entre los discursos y las prácticas, siendo los primeros inclusivos y las segundas alineadas con el tradicional sistema de medidas de atención a la diversidad. La inclusión es un movimiento alineado con las escuelas que derriban barreras para la presencia, la participación y el aprendizaje de todo el alumnado en contextos comunes. Por todo ello, es preciso avanzar propuestas para difundir el significado real de la inclusión con objeto de crear marcos compartidos como paso inicial para caminar hacia la educación inclusiva.

Palabras Clave: Equidad, inclusión educativa, exclusión, integración, formación docente. a documentary analysis of the educational administration regulations regarding inclusion. From the results obtained, it seems proved that the concept of inclusion is still not understood in ordinary centers. In addition, teachers believe that they do not have the appropriate training to respond to diversity, cast doubt the right of all students to enroll in common schools and supports the maintenance of special education centers. For its part, the analysis of the regulatory procedures of the administration shows a lack of coherence between the discourses and practices, being the first inclusive and the second one aligned with the traditional system of attention to diversity measures. Inclusion is a movement aligned with schools that break down barriers for the presence, participation and learning of all students in common contexts. Therefore, proposals must be made to disseminate the real meaning of inclusion in order to create shared frameworks as an initial step to move towards inclusive education.

Keywords: Equity, educational inclusión, exclusión, integration, teacher training.

\section{Introducción}

En estos tiempos educativos en los que tanto se habla de escuela inclusiva, tal vez resulte interesante devolver al debate a su antónimo, la exclusión educativa (González et al., 2016). Como ciudadanos/as y como docentes, diariamente somos testigos de procesos de exclusión educativa en vivo. Vemos a niños y niñas que se montan en transportes escolares para ir a centros segregados en función de su capacidad. Alumnado que entra junto en edificios comunes pero que luego se concentran durante parte de la jornada lectiva en aulas distintas, donde se les proporcionan los refuerzos y apoyos que se diseñan con el objetivo de superar su desfase. Adolescentes sometidos a medidas de atención a la diversidad como PMAR (Programa de Mejora del Aprendizaje y el Rendimiento), desdobles, agrupamientos flexibles y demás terminología específica que, revestida de enjundia semántica, enmascara su carácter de tirita pedagógica exclusiva o excluyente. Tal vez lo más singular de estas medidas es que se justifican por el bien del alumnado, como si fuese preciso, desde un enfoque asistencialista y caritativo (Zambrano, 2019), ayu- 
dar a alguien a ser como los demás, como los normales. Esto devuelve de nuevo el debate educativo al análisis de los y las destinatarios/as de la inclusión. Esto es, pone el foco sobre si son los excluidos, los apartados, los diferentes, los únicos receptores de las políticas educativas inclusivas y de dónde viene esa idea en nuestro sistema educativo.

En la década de los 90 del siglo pasado, España se encontraba en pleno movimiento de renovación pedagógica. La promulgación de la LOGSE y el concepto de integración estaban revolucionando el enfoque educativo con el que atender al alumnado con capacidades diferentes, renombrado en aquel momento como con necesidades educativas especiales (Martínez, 2002). Probablemente, puede afirmarse que ahí nació, en nuestro peculiar ecosistema educativo, el concepto de inclusión educativa. La Declaración Mundial sobre Educación para Todos y el Marco de Acción para Satisfacer las Necesidades Básicas de Aprendizaje, aprobados durante la Conferencia Mundial sobre Educación para Todos celebrada en Jomtien, Tailandia, en marzo de 1990 (https://www.oei.es/historico/efa2000jomtien.htm), dejó claro que el camino educativo a seguir no podía ser otro que las escuelas para todos y todas, sin exclusiones. Cuatro años después, en 1994, la Declaración de Salamanca (http://www.declaraciondesalamanca.org/) reafirmó el enfoque inclusivo como único futuro de la educación. Consecuentemente, 92 gobiernos y 25 instituciones internacionales reconocieron "la necesidad y urgencia de impartir enseñanza a todos los niños, jóvenes y adultos con necesidades educativas especiales dentro del sistema común de educación". A través del Marco de Acción publicado, instaban a los gobiernos a luchar contra la exclusión con medidas como la de la transformación de los centros de educación especial en centros de recursos y formación. Transcurridos 25 años desde entonces, éstos continúan sin ser una realidad generalizada en nuestro país.

Como viene siendo norma en el sistema educativo español: nuevo gobierno, nueva ley de educación. La Ley Orgánica de Educación (LOE) en 2006, popularizó el término de inclusión al aparecer en su desarrollo normativo como principio a seguir en sustitución del concepto de integración. La confusión entre ambos conceptos es constatable en la literatura científica (Muntaner, 2010; Arenas, 2016), siendo relativamente frecuente encontrar en los centros educativos opiniones diversas respecto a lo que se consideraba una vuelta de tuerca del principio de integración cuando aún no se encontraba asentado. Además del fundamento de integración, la LOE acuñó el concepto de alumnado con necesidad específica de apoyo educativo (NEAE). Ahora se distinguía, además de al alumnado con necesidades educativas especiales (por discapacidad o trastorno grave de conducta), a aquel otro que podía precisar una atención educativa diferente a la ordinaria (Calle, 2010), por presentar altas capacidades, dificultades de aprendizaje, TDAH (Trastorno por Déficit en la Atención e Hiperactividad, concepto luego incluido en la Ley Orgánica para la Mejora de la Calidad Educativa, LOMCE, 2013), incorporación tardía al sistema educativo español u otras condiciones personales o de historia escolar. De este modo, se definía claramente al colectivo de alumnado susceptible de ayuda pedagógica especial en virtud de sus condicionantes 
personales. Todo ello ha derivado en un proceso de etiquetado extremo con objeto de organizar un dispositivo burocrático de dispensa de recursos humanos, materiales y curriculares que parece no tener fin.

En el ámbito de la investigación, ya desde la década de los 90 teóricos como Skrtic (1991), alertaban sobre el peligro de que la inclusión se convirtiera en más de lo mismo (más Educación Especial), si su discurso no atacaba aspectos claves para el cambio. Analizar las instituciones escolares, su organización y sus prácticas, se señalaba, era una acción primordial para poder implementar políticas inclusivas. Sin embargo, las administraciones educativas optaban por dotar a los centros de recursos especializados para tratar de normalizar las diferencias. Esta forma de proceder aventuraba que las políticas de inclusión serían asimiladas de la misma manera que las integradoras (Skrtic, Sailor \& Gee, 2005), y se convertirían en una forma de pragmatismo ingenuo, en un modo de analizar y resolver los problemas irreflexivamente (Artiles \& Kozleski, 2016). Otro ejemplo a tener en cuenta es el de Mary Warnock, autora del famoso informe popularizado con su mismo nombre (Warnock, 1981) que ha pasado a la memoria bibliográfica educativa como el embrión del concepto de necesidades educativas especiales. La misma Mary Warnock recogió, cuatro años después, sus críticas a su propio concepto, tachándolo de falsamente objetivo y endeble, conceptualmente hablando (Slee \& Weiner, 2001). Más allá fue Len Barton (1987) al afirmar que las necesidades educativas especiales encerraban el eufemismo del fracaso de las escuelas.

Aunque estas alertas, que deberían guiar el camino de la transformación educativa hacia sistemas más equitativos, están presentes a lo largo de los años, lo cierto es que la dirección a tomar en este camino no parece clara. La confusión entre los conceptos de integración e inclusión (González \& Cano, 2013) es una constante, muy frecuente. A ello se une la problemática de los recursos humanos destinados para el alumnado con necesidades educativas especiales. Así, es necesario recordar que la integración supuso un paso más frente a la segregación escolar para el alumnado con discapacidad (Vicente, 2009), mientras que la inclusión aboga por crear escuelas y aulas para todo el alumnado (Muntaner, 2017). Reducir el concepto de inclusión a una cuestión de recursos humanos especializados para el alumnado con necesidades es un error simplista y deja fuera de la ecuación múltiples factores culturales y sociales de gran importancia. Sin cuestionar la necesidad de contar con suficientes recursos humanos, materiales y fungibles, la inclusión educativa no se implementa sólo con tener más docentes y más presupuesto. Hay que partir de conocer profundamente el concepto de inclusión. La idea de escuela inclusiva (Ainscow, 2017) que tenga el profesorado es determinante para desarrollar la educación inclusiva. Así pues, preguntar al profesorado sobre su opinión acerca de la educación inclusiva parece determinante para la implementación de la escuela inclusiva. De igual modo, es necesario comprobar si realmente se acompañan los discursos políticos y normativos con acciones congruentes. Desde estos presupuestos, y tomando la educación básica en Asturias como contexto de análisis, la intención de esta investigación se centró describir si tanto el profesorado como los responsables de la administración educativa conocen el significado del concepto de inclusión educativa. El propósito era interpretar si las posibles repercusiones de las concepciones existentes en las prácticas de am- 
bos colectivos tal vez estuvieran contribuyendo a consolidar la arquitectura de la exclusión. En consecuencia, colaborando en los esfuerzos comunitarios por implementar verdaderas escuelas inclusivas (González 2012; Torres González \& Castillo Arredondo, 2016), desarrollamos una investigación-acción participativa (Bertomeu \& Puig, 2015, Efron \& Ravid, 2019) con el claro objetivo de determinar si el profesorado ordinario conoce el significado del concepto de inclusión. La fuente de referencia para obtener esa información fue doble, por un lado se analizaron las narrativas del profesorado de Audición y Lenguaje y Pedagogía Terapéutica, perfiles profesionales que dedican su jornada laboral a trabajar con alumnado con NEAE y con el profesorado generalista. Por otro lado, para conocer directamente la opinión del profesorado generalista, no especializado en respuesta a la diversidad, la investigación se complementó con la recogida de información por parte del profesorado de Educación Secundaria Obligatoria y Formación Profesional que recibían un curso de formación al haber superado un concurso-oposición (Hernández-Amorós et al., 2017). Previo a esta recogida de datos, se realizó un análisis documental de la normativa reguladora y los procedimientos administrativos de la Consejería de Educación, que pudieran tener incidencia en el logro de la inclusividad efectiva en el contexto autonómico y en la coherencia entre la norma y su aplicación.

\section{Material y método}

Desde esta perspectiva, el objetivo principal de ta investigación fue acceder directamente al colectivo docente y recabar su percepción acerca de la educación inclusiva. Teniendo en cuenta las dimensiones de la investigación y la necesidad de obtener una representatividad lo más diversa y completa posible, se abordó al colectivo de maestras y maestros de Educación Infantil y Primaria de las especialidades de Audición y Lenguaje (AL) y Pedagogía Terapéutica (PT). Ambas disciplinas están especializadas en la respuesta a la diversidad y unidas en el trabajo diario con el alumnado en riesgo de exclusión. Por otro lado, se recabó también la opinión del profesorado generalista de Educación Secundaria Obligatoria y Formación Profesional. Respecto al primer colectivo, el especializado, se crearon varios grupos de discusión online (Ibáñez, 2016), empleando la aplicación WhatsApp (Scribano, 2017). Un total de 41 profesoras de estas especialidades intercambiaron información sobre sus competencias docentes y sobre la idea de inclusión/ integración que tenían. En el análisis de datos se empleó la metodología de las historias de vida (Bolívar, 2014) para captar descriptivamente las voces de las docentes en el contexto de su actividad profesional. El propósito perseguido era conocer su opinión respecto a cómo es entendida hoy en los centros escolares de Educación Infantil y Primaria la inclusión educativa: si como algo que tiene que ver con todas las personas o si existen sesgos que definan en la percepción y en la práctica la respuesta a la diversidad.

En cuanto al profesorado generalista de las etapas de Educación Secundaria Obligatoria y Formación Profesional, se optó por diseñar un breve cuestionario de preguntas dicotómicas recogiendo tres cuestiones clave (tabla 1). El cuestionario se administró a 299 docentes de nueva incorporación al 
sistema público de educación en el marco de la formación obligatoria a la que deben asistir una vez que han superado el concurso-oposición. Esta formación incluía una sesión de tres horas de duración sobre educación inclusiva que se inició con el cuestionario como toma de contacto con el tema.

Tabla 1: Cuestionario dirigido al profesorado de ESO y FP.

\begin{tabular}{|c|c|c|c|}
\hline & $\mathrm{Si}$ & No & Dudas \\
\hline \multicolumn{4}{|c|}{$\begin{array}{l}\text { P1: Todo el alumnado, independientemente de sus competencias cognitivas o } \\
\text { procedimentales debe escolarizarse en centros ordinarios }\end{array}$} \\
\hline \multicolumn{4}{|c|}{$\begin{array}{l}\text { P2: Como docente generalista no tengo la formación adecuada para atender en } \\
\text { el aula a alumnado con capacidades mentales o físicas limitadas }\end{array}$} \\
\hline P3: Los centros de educación especial son necesarios & & & \\
\hline
\end{tabular}

La primera cuestión (P1) enlaza con la conceptualización de la educación inclusiva como derecho, plenamente reconocido en el artículo 24,2 de la Convención Internacional de los Derechos de las personas con Discapacidad. Este derecho es incompatible con la existencia de centros segregados de educación especial, lo que liga esta cuestión con las preguntas P1 y P3.

La segunda cuestión (P2) analiza la autopercepción sobre la propia formación docente para la atención a la diversidad. Aunque la especialización sobre la diversidad se concreta en los perfiles docentes de Audición y Lenguaje, Pedagogía Terapéutica y Orientación Educativa, el cambio de paradigma sobre la inclusión traslada también la necesidad de formarse para la diversidad a los/as docentes generalistas.

La última cuestión (P3) trata de situar al profesorado ante su propia conceptualización de la educación inclusiva planteándole emitir una respuesta clara a su idea de inclusión.

Puesto que el profesorado debía mostrar su acuerdo o desacuerdo con cada cuestión, se incorporó la opción Dudas, también como respuesta debido al valor añadido que suponía esta elección para el análisis posterior.

\section{Resultados}

De las 41 docentes participantes de Educación Primaria, 33 desarrollaban sus funciones en colegios ordinarios y 8 lo hacían en Centros de Educación Especial. En función de las características del centro, se obtuvieron resultados diferentes (Figura 1). 
Figura 1: Resultados profesorado Educación Primaria.

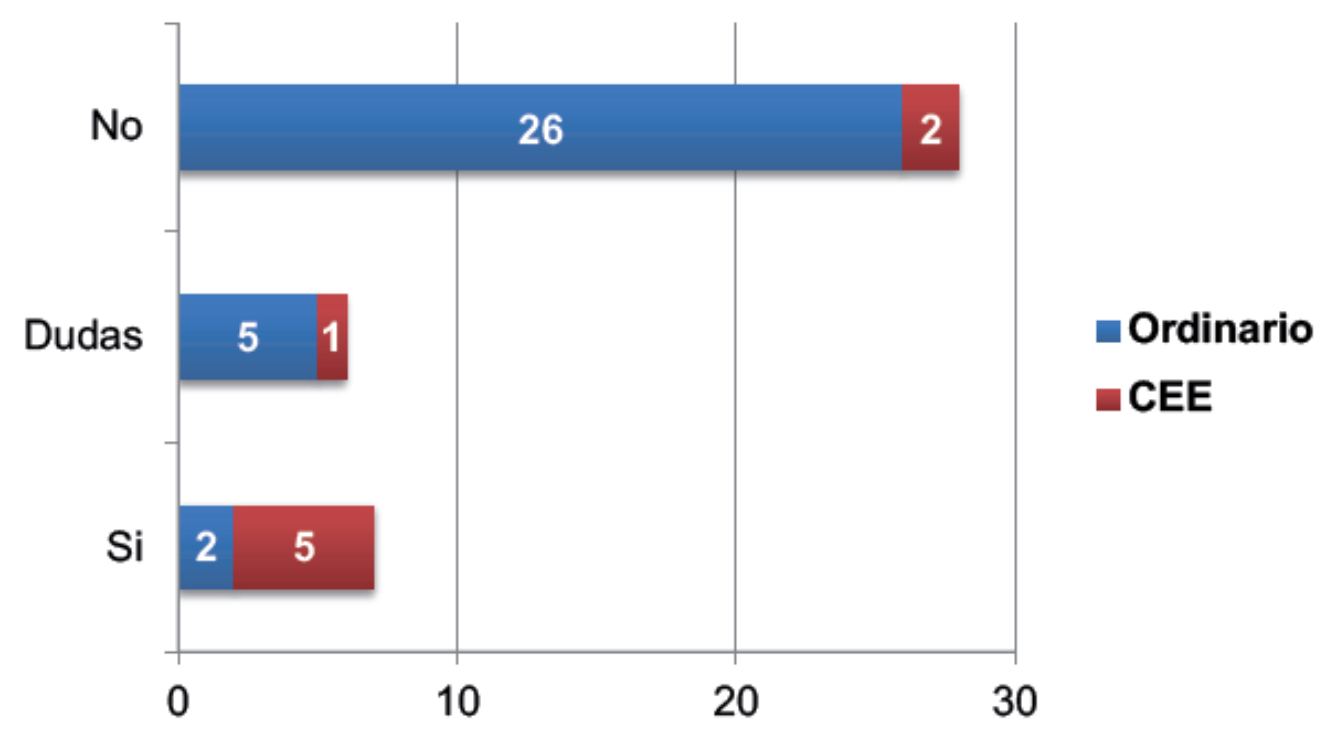

En los centros ordinarios:

- Dos maestras afirmaron que sí se entendía la inclusión como algo que tiene que ver con todas las personas, matizando una de ellas que lo entiende así porque su directora pertenece a la especialidad de Pedagogía Terapéutica y se ha esforzado en trasladar la idea correcta al claustro.

- Cinco maestras manifestaron dudas que les impedían afirmar que sí se conocía el significado de la inclusión.

- Veintiséis maestras afirmaron rotundamente que en sus centros no se comprende el concepto de inclusión, con claras referencias a la confusión entre los conceptos de integración e inclusión.

En los Centros de Educación Especial:

- Cinco maestras consideraron que en su centro sí se entendía la inclusión como algo relativo a todas las personas, alegando específicamente que estos centros escolarizan únicamente a alumnado con NEE.

- Una maestra dudó.

- Dos maestras manifestaron que no se entendía la inclusión porque siempre existe algún tipo de alumnado que no recibe la misma consideración, refiriéndose al alumnado con trastorno grave de conducta, de etnia gitana o incluso al que sensorialmente puede resultar menos agradable.

En lo que respecta a los resultados referidos al profesorado de Educación Secundaria se constata (Tabla 2) que la gran mayoría de los y las docentes consideran que los Centros de Educación Especial si- 
guen siendo necesarios y que creen que no tienen la formación adecuada para responder a la diversidad, además de manifestar muchas dudas respecto al derecho del alumnado a compartir espacios comunes.

Tabla 2: Resultados profesorado Educación Secundaria.

\begin{tabular}{|l|l|l|l|l|l|l|l|l|l|}
\hline Preguntas & \multicolumn{2}{|c|}{ P1 } & \multicolumn{3}{|c|}{ P2 } & \multicolumn{5}{|c|}{ P3 } \\
\hline Respuestas & Sí & No & Dudas & Si & No & Dudas & Si & No & Dudas \\
\hline Especialidades & 128 & 148 & 11 & 239 & 44 & 4 & 234 & 48 & 5 \\
\hline Orientación & 12 & & & 3 & 7 & 2 & 5 & 3 & 4 \\
\hline Total & 140 & 148 & 11 & 242 & 51 & 6 & 239 & 51 & 9 \\
\hline
\end{tabular}

La mayor división de opinión se obtiene en la primera afirmación, donde 148 docentes creen que no todo el alumnado tiene derecho a escolarizarse en centros ordinarios frente a 128 , que consideran lo contrario. En este sentido, se observa un importante desconocimiento de, al menos el 50\%, respecto a una de las premisas básicas de la inclusión educativa, que es el derecho a compartir lugares comunes (Figura 2).

Figura 2: Resultados a la Pregunta 1.

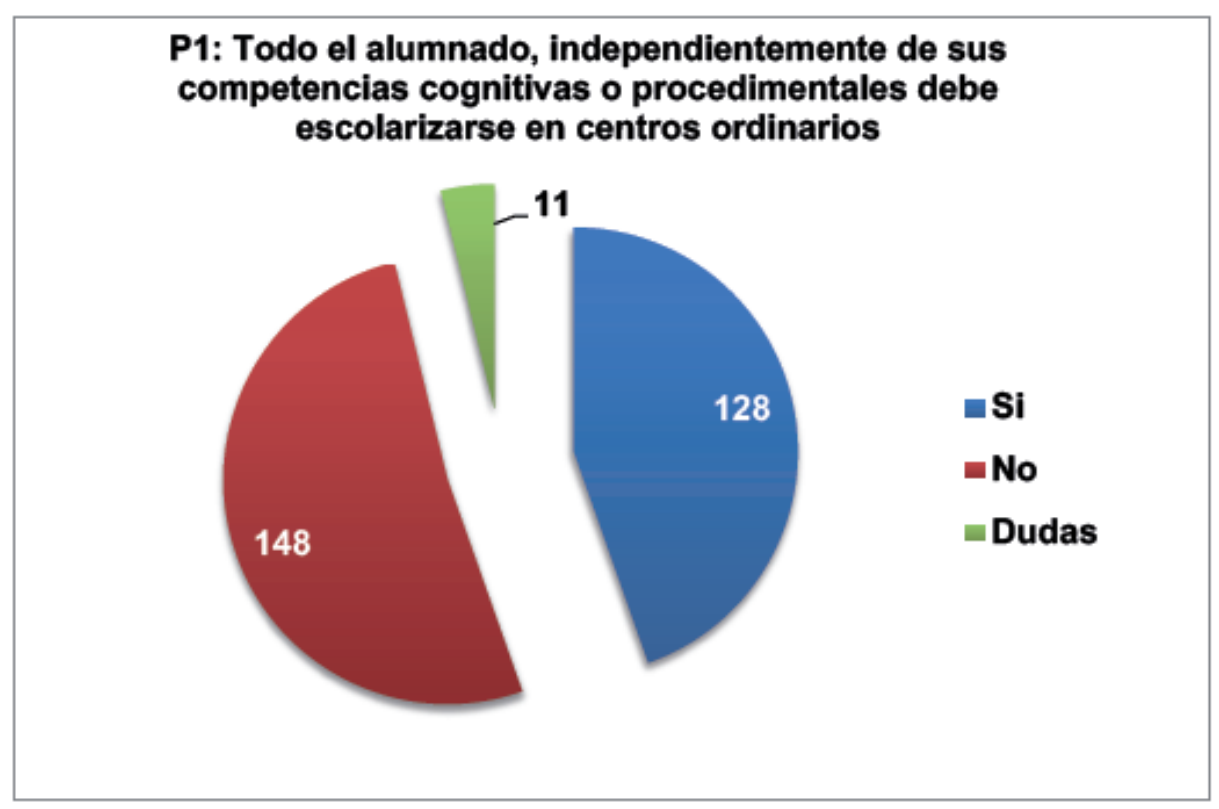

La segunda pregunta se inclina rotundamente hacia la afirmación de que el profesorado generalista considera no tener la formación adecuada para atender en el aula a alumnado diverso (Figura 3). 
Figura 3: Resultados a la Pregunta 2.

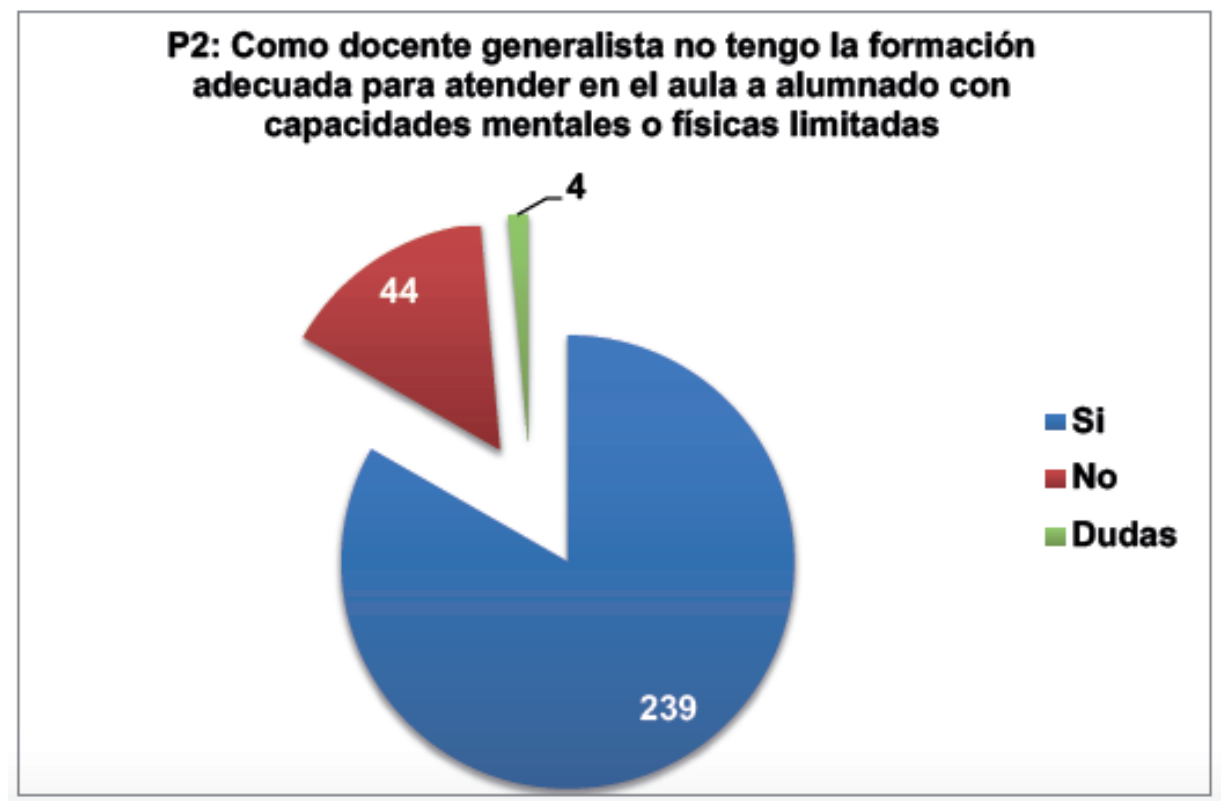

Mismo efecto se observa en las respuestas a la tercera cuestión, ya que la mayoría del profesorado considera que los centros de educación especial son necesarios. Desde una óptica rigurosa, se está afirmando que es necesaria una entidad ilegal, ya que la escolarización en modalidad específica contradice el derecho a la educación inclusiva. Parece ser que el imaginario colectivo de la mayor parte de los docentes que entran en el sistema educativo público no contempla una realidad alternativa sin centros de educación especial (Figura 4).

Figura 4: Resultados a la Pregunta 3.

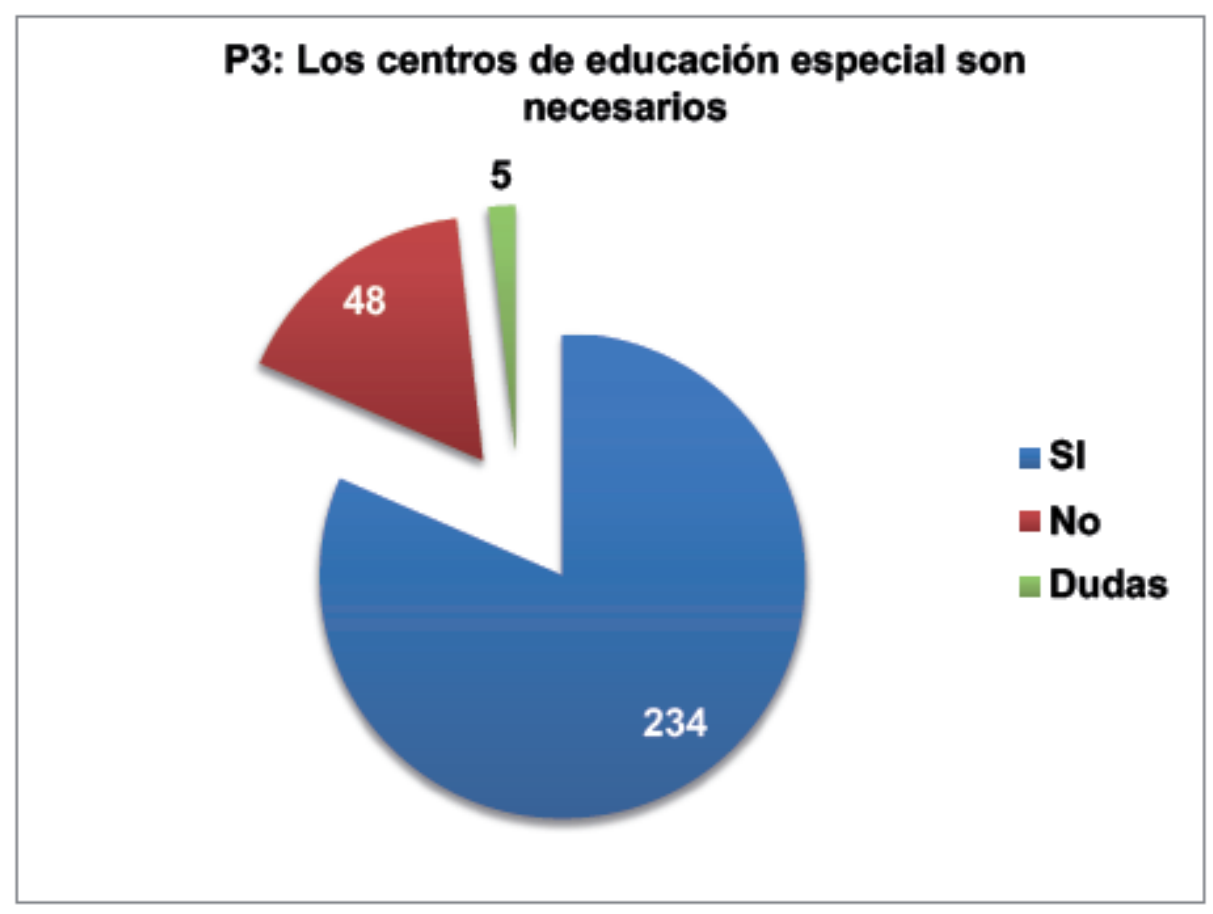


Analizando separadamente las respuestas del colectivo de Orientación educativa, se observa acuerdo en la primera pregunta: todos los orientadores y orientadoras consideran que el alumnado debe escolarizarse en centros ordinarios. Pero este acuerdo no se observa en el resto de cuestiones: dudan acerca de si estamos ante un problema de formación y se cuestionan también si los centros de educación especial son necesarios. Esta última controversia contrasta con las respuestas a la primera afirmación, observándose una contradicción manifiesta (Figura 5).

Figura 5: Resultados colectivo Orientación educativa.

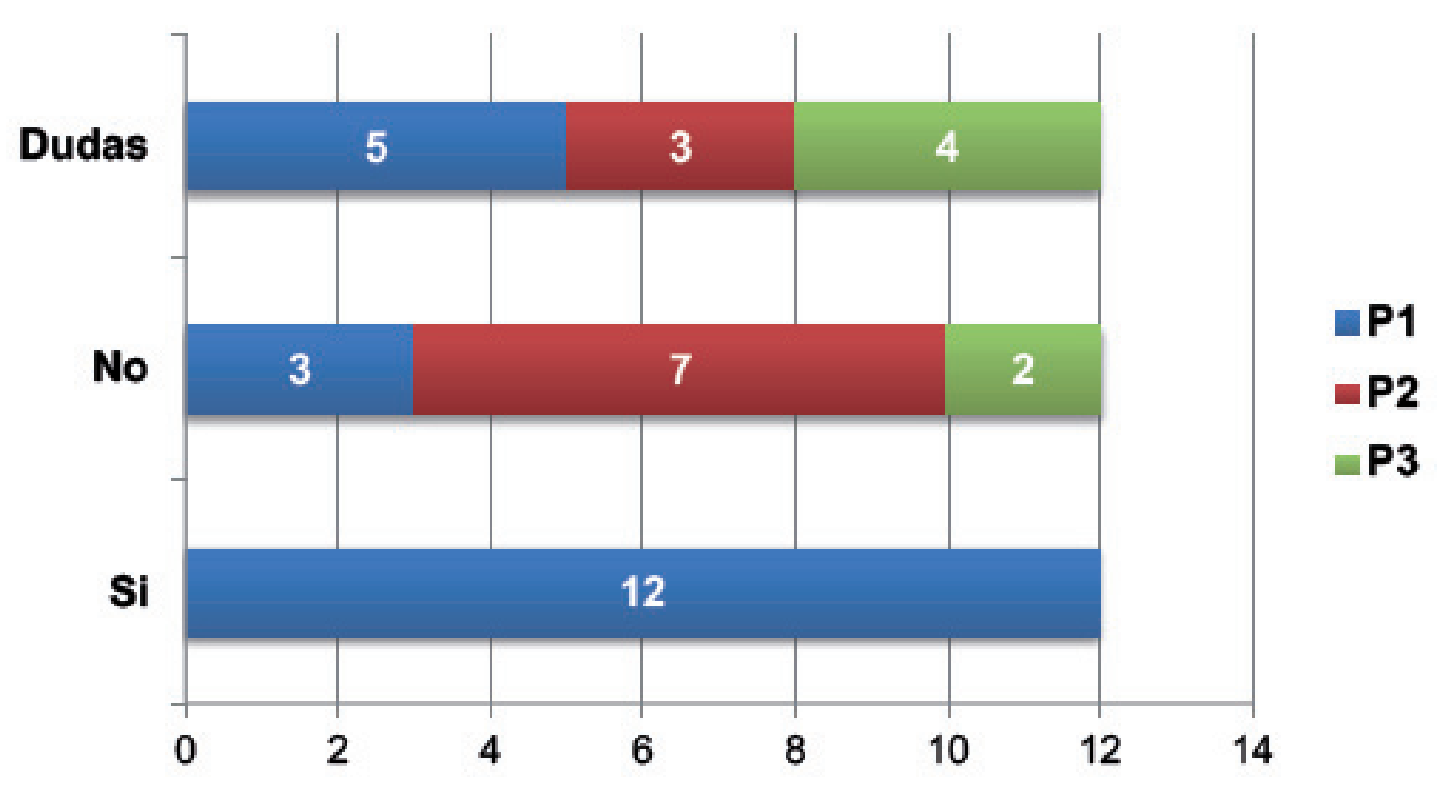

A esta parte más cuantitativa de análisis se añadió un análisis documental (Díaz-Vicario \& Gairín, 2014; Salazar-Gómez \& Tobon, 2018) respecto al grado de inclusividad que promueve la administración educativa. Esto es, a cómo se regula y normativiza la inclusión educativa en Asturias. En este sentido es llamativa la profusión de referencias a la inclusión y la equidad en los documentos institucionales: tanto en la circular de inicio de curso (comienza un nuevo curso escolar y una nueva legislatura asentada en los principios de equidad y calidad educativa...) como en las diversas instrucciones de organización y funcionamiento publicadas a lo largo del año académico (seguir avanzando en un modelo educativo que garantice la equidad, la igualdad y la inclusión educativa...).

\section{Discusión}

De ambas muestras de docentes que participaron en esta investigación se desprende que el profesorado no parece conocer el verdadero concepto de inclusión. Como se ha constatado, parecen no com- 
prender que la inclusión sea un concepto que implique vivir juntos en las aulas. Justificar esta carencia apelando a la expresión mayoritaria de carencias en la formación es arriesgado. Mas si se tiene en cuenta que quienes responden son personas que han superado un concurso-oposición para ser docentes.

De la revisión de la normativa vigente se observa la presencia de lo que Pozo et al. (2006) denominan el discurso inclusivo, siendo necesario analizar si éste se traduce y transfiere a la toma de decisiones más prácticas de la administración. No parece tal aquí. Tal vez sea porque, a pesar del discurso inclusivo, las realidades observables y procedimientos habituales siguen adoleciendo de un espíritu integrador, médico-asistencialista. Entre estas realidades y procedimientos destacan:

- La existencia de los centros de educación especial y otras modalidades segregadas de escolarización como la combinada y las aulas abiertas especializadas. Esta realidad contrasta con uno de los presupuestos básicos de la inclusión: la no segregación del alumnado en función de capacidad. Más aun, va en contra del derecho de las personas con discapacidad a la educación inclusiva, que se recoge en el artículo 24 de la Convención Internacional de los derechos de las personas con discapacidad. Ni siquiera el colectivo de docentes de la especialidad de Orientación educativa veía clara la eliminación de esos dispositivos de escolarización, lo que sugiere el estado de la cuestión acerca de la premisa de vivir juntos, que parece algo reservado para una élite del alumnado.

- La dotación de recursos humanos especializados de Audición y Lenguaje (AL), Pedagogía Terapéutica (PT) y Auxiliar Educador/a (AE). Si bien se recomienda en la normativa que estos perfiles docentes adopten roles inclusivos, es bien conocido que estos recursos se asignan a los centros en función del número de alumnado con NEE. Esta es una forma de administración de recursos obsoleta (Echeita, 2004) y llevada a cabo con una alternativa clara: la dotación básica en función del total del alumnado de los centros y su contexto sociocultural, no únicamente en función del número de alumnado con NEE. En definitiva, repartir profesionales de PT y AL únicamente en función del alumnado con NEE no hace otra cosa que difundir la creencia LOGSE de que el trabajo de estos profesionales únicamente incumbe a la respuesta directa al alumnado con discapacidad o trastorno grave de conducta.

- Para el resto de alumnado en riesgo de exclusión social se ponen en marcha itinerarios y programas tradicionales de apoyo y refuerzo como los agrupamientos flexibles y el PMAR (denominación LOMCE de los tradicionales programas de diversificación). La existencia de estas medidas de atención a la diversidad refuerzan la búsqueda de la homogeneidad a través del establecimiento de escenarios clasificadores de alumnado que buscan dividir, sin transformar profundamente, las realidades escolares. Teniendo en cuenta esta situación, es difícil que el profesorado de Educación Secundaria realice apreciaciones en pro de la convivencia de alumnado diverso, tal y como se ha reflejado en las respuestas dadas al cuestionario presentado. 
Además de las realidades descritas, centradas en la dotación de recursos y la estratificación del alumnado, la administración educativa pone en marcha otras actuaciones para remover la arquitectura de la exclusión. Son ejemplos de ello en Asturias una estrategia de Orientación para implantar el Diseño Universal del Aprendizaje (Chavarría et al., 2019), los contratos-programa para centros promotores de la equidad (Educastur, 2019) y el proyecto de nuevos espacios de aprendizaje en Educación Secundaria (Educastur, 2018). Todas ellas pueden analizarse desde un enfoque de luces y sombras, ya que si bien son iniciativas inclusivas, también son parciales y difícilmente generalizables al conjunto de centros educativos.

\section{Conclusiones}

Tras el análisis de la información cuantitativa proporcionada por el profesorado se constata que los docentes, en su amplia mayoría, siguen conceptualizado la educación inclusiva como aquella que tiene que ver con el alumnado con necesidades educativas especiales, y por extensión con el profesorado especialista en atención a la diversidad, sin sentir que sea una temática que les interpele en sus labores educadoras. Respecto al profesorado de los Centros de Educación Especial, se detecta que la cultura de apertura a la diversidad del alumnado es diferente, aunque sorprenden ciertas respuestas respecto a colectivos de alumnado como el de etnia gitana o el de trastorno grave de conducta. A nivel cualitativo, se ha evidenciado en el análisis de la normativa asturiana que el discurso inclusivo está presente, pero también se ha acreditado que las tradicionales medidas clasificatorias de atención a la diversidad siguen presentes en las prácticas e instrucciones. De este modo, la administración no contribuye a clarificar la confusión del profesorado respecto al concepto de inclusión (Lledó Carreres, 2017). Es más, puede parecer que aumenta la duda. Parece que camina hacia la inclusión en sus declaraciones de intenciones, pero posteriormente en sus procedimientos prácticos va en dirección opuesta, clasificando al alumnado según discapacidad, articulando medidas excluyentes y solicitando documentación que refuerza este modelo. De este modo el profesorado generalista confirma su creencia inicial de que la inclusión tiene que ver únicamente con el alumnado tradicionalmente catalogado con necesidades educativas y el profesorado especializado en dar respuesta a estas necesidades. En definitiva, parece que la inclusión se queda en una propuesta meramente estética, que no propone cambios sistémicos y profundos en el sistema educativo.

De unos datos y otros se desprende la necesidad de remover la arquitectura de la exclusión. Sin duda, es necesario conocer y analizar teóricamente cómo funciona esa arquitectura para poder derribar o minimizar las barreras existentes en el camino hacia la escuela inclusiva. En este sentido es importante evitar que las políticas de discriminación positiva se conviertan en segregadoras (Castell, 2004) y tratar de que la diversidad no se convierta en desigualdad. Analizando los factores sociales, culturales, políticos, administrativos y prácticos que intervienen en el proceso exclusión-inclusión, las administraciones 
y los agentes sociales necesitan desarrollar un plan de acción que parta de la construcción de una mirada compartida sobre la educación inclusiva. Trabajar colaborativamente en las instituciones, de manera intra e interinstitucional, y dando voz al profesorado y al alumnado, es indispensable para crear una cultura compartida inclusiva, asentada en valores democráticos. Probablemente de esta manera se evitara constatar que el profesorado ni comprende el significado del concepto de inclusión ni tiene clara la dirección de los movimientos que tratan de aunar equidad y calidad. Esta discrepancia entre las intenciones inclusivas de la administración y las acciones del profesorado es, como se ha visto, dilemática y multicausal. Dota de especial importancia la necesidad de que la administración educativa guie al profesorado en su camino hacia la inclusión, clarificando el término, definiendo los objetivos, ofreciendo formación de acompañamiento y re-evaluando continuamente las barreras y los facilitadores que van apareciendo.

A nuestro juicio, un plan de acción semejante debe, en primer lugar, apostar y visibilizar unas líneas de política educativa claras que aborden las barreras existentes, tratando de minimizarlas en lo posible:

- Barreras sociales y culturales, a derribar tratando de construir comunidad y apostando por la asunción compartida de valores democráticos como base para la acción.

- Barreras políticas y administrativas, que podrían minimizarse a través de miradas compartidas con el objeto de normativizar y procedimentar el camino hacia la inclusión.

- Barreras prácticas, que deberían tumbarse apostando por la formación, la implantación y el seguimiento de enfoques y metodologías que permitan la personalización del aprendizaje.

Esta apuesta necesita materializarse a través de medidas estructurales, ordinarias, adicionales y extraordinarias pensadas desde una óptica que conjugue la calidad y la equidad (Booth \& Ainscow, 2011). De todas las medidas posibles, inabordables por su extensión, se destacan aquí las tres que, a la luz de los resultados obtenidos, parecen mas urgentes:

- La acogida: dar la bienvenida y acompañar es uno de los principios básicos para posteriormente poder compartir y trabajar colaborativamente (Susinos \& Parrilla, 2013). Así, planificar la acogida al nuevo personal en las administraciones y en los centros educativos es fundamental, ofreciendo apoyo y también explicando claramente a cada trabajador o trabajadora el proyecto educativo al que se suma y la filosofía que lo respalda. Por supuesto la acogida al alumnado y a las familias también deberá ser protocolizada y concebida como una acción clave inclusiva.

- La prevención: responder a la diversidad implica desarrollar políticas preventivas y sistémicas de las dificultades de aprendizaje desde los primeros años de la escolaridad, huyendo de los modelos de espera al fracaso y apostando por modelos inclusivos de respuesta a la intervención (Jimenez, 2019). Para ello es fundamental apostar desde Educación Infantil por la práctica ba- 
sada en la evidencia, potenciando el papel contextual de la calidad de la instrucción, ya que uno de los factores más determinante del éxito académico es la calidad de la formación del profesorado. En las primeras etapas tienen además mayores oportunidades de detectar indicadores de riesgo y de implementar programas personalizados de intervención temprana orientados bajo el prisma del Diseño Universal del Aprendizaje (Pastor, 2018).

- La creación de redes: crear redes de apoyo (Abellan, 2017; Sánchez, Rodríguez \& Abellán, 2018) es fundamental para el éxito de cualquier propuesta. El sentido de pertenencia y el acompañamiento en el camino puede lograrse a través de la creación de esas redes como espacios de reflexión que podrían articularse con un carácter más interdisciplinar u homogéneo en función de necesidades concretas. Así, las redes de direcciones de centro con orientación inclusiva, las redes que apuesten por un modelo de Educación Secundaria alternativa, las redes ecosociales, etc... podrían aunarse a otras más tematizadas por especialidades (Orientación, Educación Infantil,...). Además, es imprescindible la apuesta por la apertura, incorporando a agentes externos a los centros, de cada comunidad local.

Con todo ello, remover la arquitectura de la exclusión no es tarea sencilla, ya que trasciende lo escolar y se asienta en lo social (Parrilla, 2002), pero está claro que el camino educativo pasa por intentar desarrollar procesos de transformación. Estos procesos, necesariamente colaborativos, deben partir de un conocimiento profundo de la realidad y de los distintos aspectos que intervienen en el fenómeno de la exclusión escolar y de aquellos otros que ya han sido señalados por la investigación como claves para el éxito.

\section{Referencias}

Abellán, C.M.A. (2017). Redes de colaboración entre escuelas inglesas para la mejora de la inclusión socioeducativa. Profesorado, Revista de Currículum y Formación del Profesorado, 21(2), 29-48.

Ainscow, M. (2017). Haciendo que las escuelas sean más inclusivas: lecciones a partir del análisis de la investigación internacional. Revista de Educación inclusiva, 5(1), 39-49

Arenas, A.K. (2016). De la integración a la inclusión: una escuela para todos. ARJÉ. Revista de Postgrado, 10(19), 241-249.

Artiles, A.J., \& Kozleski, E.B. (2016). Inclusive education's promises and trajectories: Critical notes about future research on a venerable idea. Education Policy Analysis Archives, 24(43). http://dx.doi.org/10.14507/epaa.24.1919 
Barton, L. (1987) The Politics of Special Educational Needs. London: Falmer Press

Bolívar, A. (2014). Las historias de vida del profesorado: voces y contextos. Revista mexicana de investigación educativa, 19(62), 711-734.

Booth T. \& Ainscow, M. (2011). Guía para la educación inclusiva: desarrollando el aprendizaje y la participación en los centros escolares. Bristol: CSIE.

Bertomeu, P.F. \& Puig, M.S. (2015). El valor del grupo dentro de una investigación acción participativa. En AIDIPE (Ed.) Investigar con y para la sociedad (Vol. 1), pp. 51-62.

Calle, R.C. (2010). La atención educativa integral a los alumnos con necesidades específicas de apoyo educativo: actitudes, valores y normas. Hekademos: Revista Educativa Digital, 7, 23-42.

Castell, R. (2004). Encuadre de la exclusión. En: S. Karsz, La exclusión: bordeando sus fronteras. Definiciones y matices. Barcelona: Gedisa.

Chavarría, E., Rosa, M., Gallego Condoy, M.B., \& González-Montesino, R.H. (2019). Diseño Universal del Aprendizaje e inclusión en la Educación Básica. ALTERIDAD. Revista de Educación, 14(2), 207-218.

Díaz-Vicario, A., \& Gairín, J. (2014). Entornos escolares seguros y saludables. Algunas prácticas en centros educativos de Cataluña. Revista Iberoamericana de educación, 66, 189-206.

Echeita Sarrionandia, G. (2004). La situación educativa del alumnado con necesidades educativas especiales asociadas a discapacidad en la Comunidad de Madrid. Psicología Educativa, $10(1), 19-44$.

González, A.E., \& Cano, A.M. (2013). Inclusión educativa y profesorado inclusivo: aprender juntos para aprender a vivir juntos (Vol. 122). Madrid: Narcea Ediciones.

González, J.A.T. (2012). Estructuras organizativas para una escuela inclusiva: promoviendo comunidades de aprendizaje. Educatio siglo XXI, 30(1), 45-70

González, M.B.A., González, M.T.G., Domínguez, B.M., Cano, J.M.N., Entrena, M.J.R., \& Ruiz, M.V. (2016). Inclusión y exclusión educativa:: Realidades, miradas y propuestas (Vol. 8). Valencia: Nau Llibres.

Hernández-Amorós, M.J., Urrea-Solano, M.E., Alós, L.G., San Martín, N.G.L., López, R.S.m \& Fernández, J.M.G. (2017). Actitudes de los maestros en formación sobre la diversidad e 
inclusión educativa. International Journal of Developmental and Educational Psycho$\log y, 4(1), 45-53$.

Ibáñez, J. (2016). Cómo se realiza una investigación mediante grupos de discusión. En M.G. Ferrando et al. (coord.) El análisis de la realidad social: Métodos y técnicas de investigación (pp. 418-434). Madrid: Alianza.

Jiménez, J. (2019). Modelo de respuesta a la intervención. Un abordaje preventivo para el abordaje de las dificultades específicas de aprendizaje. Madrid: Pirámide.

Lledó Carreres, A. (2017). Una revisión sobre la respuesta educativa de los centros escolares en el cambio hacia la inclusión educativa. Revista de Educación Inclusiva, 3(3), 1-16

Martínez, J.J.C. (2002). Educación especial e integración escolar a través del desarrollo normativo: un estudio comparado de la pluralidad estatal. Revista de educación, 327, 341-359.

Muntaner, J.J. (2010) De la integración a la inclusión: un nuevo modelo educativo. En P.Arnaiz, M.A. Hurtado, \& F.J. Soto (Coords.) 25 Años de Integración Escolar en España: Tecnología e Inclusión en el ámbito educativo, laboral y comunitario. Murcia: Consejería de Educación, Formación y Empleo.

Muntaner, J.J. (2017). Prácticas inclusivas en el aula ordinaria. Revista de Educación Inclusiva, 7(1).

Parrilla, A. (2002). Acerca del origen y del sentido de la educación inclusiva. Revista de Educación, $337,11-29$.

Pastor, C. A. (2018). Diseño Universal para el Aprendizaje un modelo didáctico para proporcionar oportunidades de aprender a todos los estudiantes. Padres y Maestros/Journal of Parents and Teachers, 374, 21-27.

Pevida, D. (coord). (2018): Nuevos Espacios de aprendizaje. Oviedo: Serie Innovación pedagógica, Consejería de Educación y Cultura.

Pozo, J.I., Scheuer, N., Pérez Echeverría, M.P., Mateos, M., Martín, E. \& de Cruz, M. (2006). Las concepciones de profesores y alumnos sobre el aprendizaje y la enseñanza. Barcelona: Editorial Graó.

Scribano, A. (2017). Miradas cotidianas. El uso de Whatsapp como experiencia de investigación social. Revista Latinoamericana de Metodología de la Investigación Social, 13, 8-22. 
Skrtic, T.M. (1991). The Special Education Paradox: Equity as the way to Excellence. Harvard Educational Review, 61(2), 148-206.

Skrtic, T., Sailor, W., \& Gee, K. (2005). Voice, collaboration, and inclusion: Democratic themes in educational and social reform initiatives. En: D. Mitchell (ed.), Systems and Contexts, Vol. 1 (pp 214-226). London: Routledge Farmer

Slee, R. y Weiner, G. (2001). ¿Eficacia para quien? Crítica de los movimientos de las escuelas eficaces y de la mejora escolar. Madrid: Akal Ediciones.

Salazar-Gómez, E., \& Tobon, S. (2018). Análisis documental del proceso de formación docente acorde con la sociedad del conocimiento. Revista Espacios, 39(53)

Sánchez, P.A., Rodríguez, R.D.H., \& Abellán, C.M.A. (2018). Redes de apoyo y colaboración para la mejora de la educación inclusiva. Profesorado, Revista de Currículum y Formación del Profesorado, 22(2), 29-49.

Susinos, T. \& Parrilla, A. (2013). Investigación inclusiva y tiempos difíciles. Certezas provisionales y debates pendientes. Revista Iberoamericana sobre Calidad, Eficacia y Cambio en Educación, 11(2).

Torres González, J.A., \& Castillo Arredondo, S. (2016). Incidencia de las políticas de apoyo educativo en las estructuras organizativas de los centros desde la perspectiva del profesorado: Un estudio en la provincia de Jaén. Educación XXI: Revista de la Facultad de Educación, 19(2), 205-228.

Vicente, J.A.L. (2009). Perspectiva legal de la Educación Especial en España (1970 2007): hacia la plena integración educativa y social de las personas con discapacidad. En: El largo camino hacia una educación inclusiva: la educación especial y social del siglo XIX a nuestros días: XV Coloquio de Historia de la Educación, Pamplona-Iruñea, 29, 30 de junio y 1 de julio de 2009 (pp. 495-510). Navarra: Universidad Pública de Navarra.

Warnock, M. (1981). Meeting Special Educational Needs, London: Her Britannic Majesty's Stationary Ofice,

Zambrano, J.R. (2019). Reflexiones epistemológicas para la formación de educadores especiales. Educação em Foco, 24(3), 807-826. 